\title{
CORRESPONDENCE
}

\section{Reply to "Comments on 'Reconstruction of the Gulf Stream from 1940 to the Present and Correlation with the North Atlantic Oscillation"”}

\author{
Sylvain WATElet, JEAN-MARIE BECKERS, AND ALEXANDER BARTH \\ Department of Astrophysics, Geophysics and Oceanography, and GeoHydrodynamics and Environment \\ Research Unit, and Interfacultary Center for Marine Research, \\ University of Liège, Liège, Belgium
}

(Manuscript received 8 August 2019, in final form 12 September 2019)

We thank Lequan Chi, Sultan Hameed, and Christopher L. P. Wolfe for the thorough analysis of our work on the Gulf Stream North Wall (Chi et al. 2019). In particular, their critical assessment of our mathematical definition of the northern boundary compared to their established analysis of this position is something we could have investigated in more detail. Our major objective was, however, not so much to define the position but to check whether or not a correlation with NAO could be detected; in other words we were most interested in changes of the position rather than in the position itself. To verify how sensitive our results are with respect to the position's definition, we performed some sensitivity tests. In our paper, the position $p_{1}$ was calculated using the fit of the SST by an error function described in the paper as Eq. (3.1) (Watelet et al. 2017). Hereafter are described our four sensitivity tests.

1) S1-Global shift of the position

$$
p^{\prime}=p_{1}-40 .
$$

Applied before EOF calculations. This should only slightly modify the EOF-based index.

2) S2-Taking out the time average \langle\rangle in each latitude

$$
p^{\prime}=p_{1}-\left\langle p_{1}\right\rangle \text {. }
$$

This takes out for each longitude the time average before calculating the EOFs, one of the typical

\footnotetext{
Corresponding author: Sylvain Watelet, swatelet@uliege.be
}

operations performed before calculating EOFs (Jolliffe 2002).

3) S3-Taking out a seasonal cycle

$$
p^{\prime}=p_{1}-p_{s} .
$$

Instead of subtracting the time average, we can subtract for each month the climatological average of this month $p_{s}$ to extract the nonseasonal signal before the EOF calculations and get yet another time variability of the index.

4) S4-Moving the position to the southern side of the sigmoid fit

$$
p^{\prime}=p_{1}-\alpha p_{4}
$$

For each longitude we now use the position where the gradient starts instead of the position of the maximum gradient by subtracting a fraction of the thickness of the "wall." That would be consistent with the graphs provided in the comments on our paper. Note that this introduces changes both in space and time as $p_{4}$ is also fitted in each longitude and month. The parameter alpha can be chosen so as to have an average position close to the one depicted in the comment paper. With a value of $\alpha=1$, we get the average position shown in our Fig. 1, to be compared to Fig. 3 of Chi et al. (2019). Similarly to S3, the seasonal cycle is taken out as well.

It turns out that for the four sensitivity tests (S1-S4) the calculated GSNW indexes are changed but are highly correlated with the original one (see Fig. 2 showing the modified indexes and 


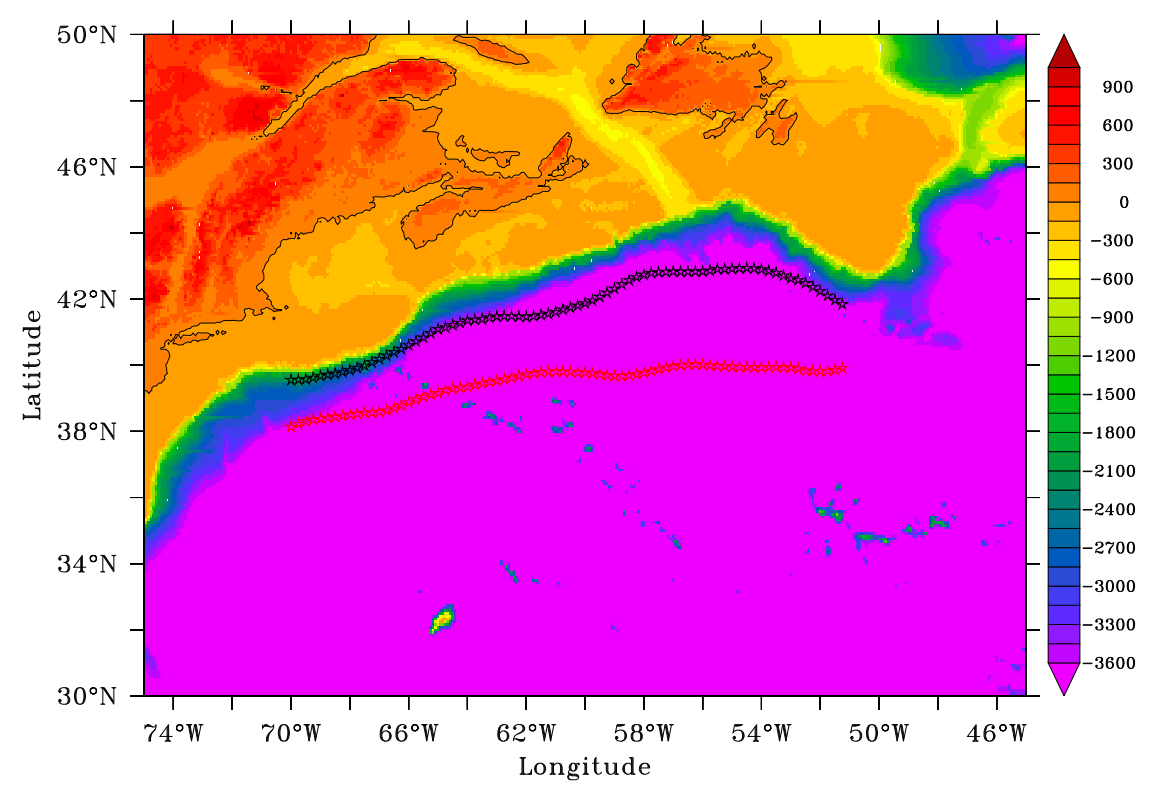

FIG. 1. Bathymetry (m) in the GS region and average GSNW position using S0 (black) and S4 (red) definitions between 1940 and 2014.

corresponding correlations with the original index S0 in Table 1).

The correlation with the Taylor index between 1982 and 2014 is somewhat decreased (from 0.4331 in S0 to 0.3482 in S1, 0.3824 in S2, 0.3658 in S3, and 0.3427 in S4, still close to a significant level). But as already clearly stated in our original paper, it is difficult to compare those indexes directly because of their different regional extent. The correlations with NAO remain very stable and even slightly increase with the S4 definition (see Table 2). In every case, the correlation NAO-GSNW peaks

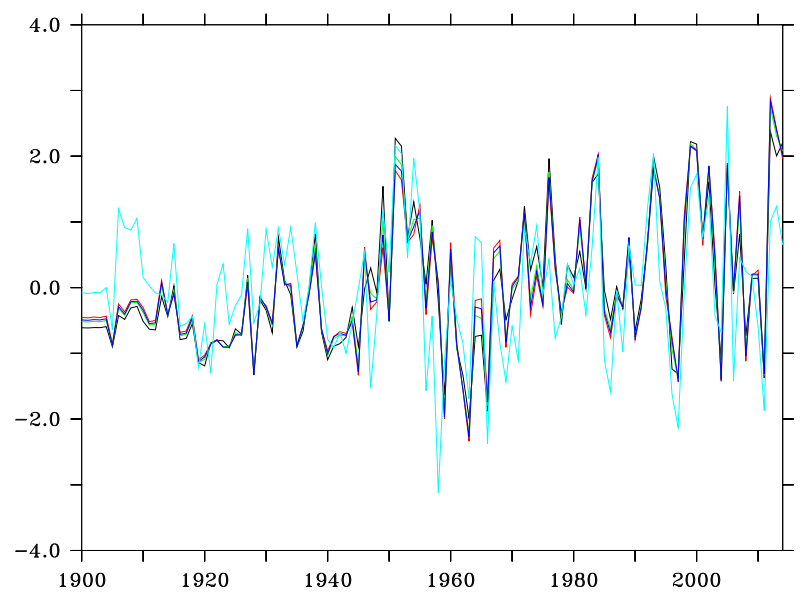

FIG. 2. GSNW sensitivity tests. The indices are performed from S0 (black), S1 (red), S2 (green), S3 (blue), and S4 (light blue) definitions. at a 1-yr time lag. For completeness we thus updated the web page with the indices included in this modified version (https://swatelet.github.io/ \#gs-indexes).

In summary, the main conclusions of our paper seem to be robust with respect to the actual definition of the GSNW position, but an adaptation of the mathematical definition we used should be done if the aim is to be consistent with the Chi et al. (2019) definition. We provided one such adaptation in the present reply, but there is certainly room for a

TABLE 1. Correlations between GSNW indices performed from definitions S1, S2, S3, and S4 and the GSNW index from S0 definition.

\begin{tabular}{ccccc}
\hline & S1 & S2 & S3 & S4 \\
\hline 1940-2014 & 0.9495 & 0.9791 & 0.9644 & 0.7607 \\
$1960-2014$ & 0.9602 & 0.9822 & 0.9708 & 0.7908 \\
\hline
\end{tabular}

TABLE 2. Correlations between GSNW indices from S0, S1, S2, S3, and S4 definitions and the Hurell NAO index with or without time lag (NAO preceding GS).

\begin{tabular}{cccc}
\hline \hline 1940-2014 & NAO $(0 \mathrm{yr})$ & NAO $(-1 \mathrm{yr})$ & NAO $(-2 \mathrm{yr})$ \\
\hline S0 & 0.1812 & 0.3692 & -0.02329 \\
S1 & 0.1425 & 0.2954 & -0.07151 \\
S2 & 0.1604 & 0.3187 & -0.05656 \\
S3 & 0.1517 & 0.3043 & -0.06479 \\
S4 & 0.2778 & 0.3985 & 0.07118 \\
\hline
\end{tabular}


more subtle adaptation, possibly taking into account information on shelf-water boundaries in the fit and the resulting definition of the GSNW position.

\section{REFERENCES}

Chi, L., S. Hameed, and C. L. Wolfe, 2019: Comments on "Reconstruction of the Gulf Stream from 1940 to the
Present and Correlation with the North Atlantic Oscillation." J. Phys. Oceanogr., 49, 2731-2734, https://doi.org/10.1175/ JPO-D-19-0096.1.

Jolliffe, I., 2002: Principal Component Analysis. Springer, $488 \mathrm{pp}$.

Watelet, S., J.-M. Beckers, and A. Barth, 2017: Reconstruction of the Gulf Stream from 1940 to the present and correlation with the North Atlantic Oscillation. J. Phys. Oceanogr., 47, 2741-2754, https://doi.org/10.1175/JPO-D17-0064.1. 